\title{
Geodesic modes driven by plasma fluxes during oblique NB heating in tokamaks
}

F. Camilo de Souza, A. G. Elfimov, and R. M. O. Galvão

Citation: Physics of Plasmas 25, 122507 (2018); doi: 10.1063/1.5039729

View online: https://doi.org/10.1063/1.5039729

View Table of Contents: http://aip.scitation.org/toc/php/25/12

Published by the American Institute of Physics

\section{Articles you may be interested in}

Erratum: "Asymmetric diffusion of cosmic rays" [Phys. Plasmas 22, 091504 (2015)]

Physics of Plasmas 25, 129901 (2018); 10.1063/1.5082558

Cutoff frequencies of accelerating modes propagation inside a cylindrical waveguide filled with radially inhomogeneous magnetized plasma

Physics of Plasmas 25, 122105 (2018); 10.1063/1.5065903

Radial density and heat fluxes description in the velocity space: Nonlinear simulations and quasi-linear calculations

Physics of Plasmas 25, 122304 (2018); 10.1063/1.5057420

Hybrid simulations of fishbone instabilities and Alfvén eigenmodes in DIII-D tokamak

Physics of Plasmas 25, 122504 (2018); 10.1063/1.5064647

High power gamma flare generation in multi-petawatt laser interaction with tailored targets

Physics of Plasmas 25, 123105 (2018); 10.1063/1.5062849

Particle-in-cell simulations of Hall thruster acceleration and near plume regions

Physics of Plasmas 25, 123504 (2018); 10.1063/1.5054009 


\title{
Geodesic modes driven by plasma fluxes during oblique NB heating in tokamaks
}

\author{
F. Camilo de Souza, A. G. Elfimov, and R. M. O. Galvão \\ Institute of Physics, University of São Paulo, São Paulo 05508-090, Brazil
}

(Received 10 May 2018; accepted 23 November 2018; published online 13 December 2018)

\begin{abstract}
Some relevant aspects of the instability of Geodesic Acoustic Modes (GAMs) driven by Neutral Beam (NB) injection are studied, in particular its dependence on the injection direction, that is, coor counter-injection, and on the pitch angle distribution of the beam particles in velocity space. In this paper, we further investigate these and other related issues considering the excitation of GAMs by energetic ions created during NB injection and modeled by a bump-on-tail distribution function with a sharp Gaussian dependence over the pitch angle at the injection angle. The bump is considered to have an energetic ion tail with temperature of the order of the third part of the critical energy that appears due to the slowing down effect on electrons. It is found that the maximum frequency of the GAM instability stays below the particle circulation frequency at the critical energy, and it is substantially reduced to be closer to parallel injection conditions. The instability may be preferentially driven for counter NB injection due to the interaction of plasma rotation and/or diamagnetic drift with electron current velocity. Published by AIP Publishing.
\end{abstract}

https://doi.org/10.1063/1.5039729

\section{INTRODUCTION}

Geodesic Acoustic Modes (GAMs) are $\mathrm{M}=0$ and $\mathrm{N}=0$ axisymmetric modes combined with $\mathrm{M}= \pm 1$ and \pm 2 poloidal side-bands, with frequency $\omega_{G}^{2} \approx\left(7 T_{i} / 2+2 T_{e}\right) / R_{0}^{2} m_{i}$, driven by electron and anisotropic ion pressure perturbations. ${ }^{1}$ In this expression, $T_{e}$ and $T_{i}$ are the electron and ion temperatures in energy units, respectively, $R_{0}$ is the major radius of the plasma column, and $m_{i}$ is the ion mass. These modes may drive anomalous plasma transport and energetic ions loss, as it has been theoretically established ${ }^{2,3}$ and experimentally observed. ${ }^{4-14}$ Furthermore, they may be useful as a diagnostic tool to indicate the L-H confinement transition $^{4-6}$ in tokamaks. Eigenmodes in the geodesic frequency range have been experimentally observed for a wide range of tokamak ohmic discharges ${ }^{7,8}$ and were also detected in ion cyclotron resonance (ICR) ${ }^{9}$ and Neutral Beam (NB) heating discharges. ${ }^{10-14}$ In the latter case, the instability is preferentially driven by counter injection in comparison to coinjection, especially during the current rump-up. ${ }^{10-14}$

According to the early theoretical models, in discharges with NB injection, the GAM instability can be driven due to an inhomogeneous dependence on the pitch angle distribution $^{15-17}$ in velocity space. For the parallel balanced injection, ${ }^{17}$ the inverse Landau damping is found to be the origin of instability, but a cold beam model ${ }^{18,19}$ of the bump may also reproduce the GAM instability that is named ${ }^{18}$ as reactive and it begins without threshold due to the absence of dissipation. Generally, the energetic ion model was used in the form of a slowing down distribution $F_{h} \propto n_{h} /\left(v^{3}+V_{\mathrm{cr}}^{3}\right)$, where $V_{\mathrm{cr}}=\sqrt{2 E_{\mathrm{cr}} / m_{b}} \approx 5.5 A_{h}^{1 / 3} \sqrt{T_{e} / m_{b}}$ is defined ${ }^{20}$ by the critical energy $E_{\mathrm{cr}}\left(m_{b}\right.$ is the mass of the beam particles, $A_{h}$ is the mass number, and $n_{h}$ is the fractional density of the energetic particles). Two kinds of geodesic modes were found, namely, the standard GAM and the geodesic mode driven by energetic particles (EGAM), which may appear above some critical energetic ion density. It should be noted that numerical simulations using the TRANSP code ${ }^{21}$ show that the energetic ions" "bump on tail" is formed at the critical velocity, where the GAM phase resonance may occur, and that accounting for the energetic ion temperature may affect the GAM dispersion and instability criteria.

In this work, we analyze the effect of a minor concentration of energetic ions, with velocity distribution produced by NB injection, on the GAM spectrum using a fully kinetic description for the energetic particles, while the basic plasma particles are treated in the fluid approximation. This model is based upon the following simplifying assumptions: large values of the safety factor $q$, namely, $q^{2} \gg 1$ and frequency band characterized by $v_{T e} / R q \gg \omega_{G A M} \gg v_{T i} / R q$. In comparison to previous investigations, ${ }^{15,16}$ the new features in our model are to consider the situation in which the GAM phase resonance may appear at the critical velocity where the energetic ions form the bump that begins to be substantially diffused/scattered, having the parallel temperature $T_{h} \approx E_{\mathrm{cr}} / 3$, and the difference in the physics of the modes driven by balanced and co/counter injection is under discussion.

\section{THEORETICAL MODEL FOR GAM DRIVEN BY THE BEAM}

The kinetic treatment of the GAM type modes driven by $\mathrm{NB}$ injection in large aspect ratio tokamaks, i.e., $R_{O} \gg a$, where $a$ is the minor radius of the plasma column, with circular surfaces $\left(R=R_{0}+r \cos \vartheta\right.$ and $\left.z=r \sin \vartheta\right)$, is carried out using the quasi-toroidal set of coordinates $(r, \vartheta, \zeta)$, which are formed by the magnetic field with toroidal and poloidal components, such as $B_{\zeta}=B_{0} R_{0} / R$ and $B_{\vartheta}=r B_{\zeta} / q R_{0}$, where $B_{O}$ is the value of the magnetic field at the magnetic axis. 
The simplified drift kinetic equation ${ }^{22-24}$ for any species $\alpha$ (electron, energetic, or main ions), for electrostatic oscillations, is written in the plasma species coordinate frame as

$$
\begin{aligned}
(w & \left.+v_{0 \alpha}\right) \frac{\partial f_{\alpha}}{\partial \vartheta}-\mathrm{i} \Omega f_{\alpha} \\
= & \frac{e_{\alpha}}{m_{\alpha}}\left[\frac{\left(2+\eta_{\alpha}\left(\mathrm{u}^{2}-3\right)\right)}{2 k_{0} v_{T \alpha} \omega_{c \alpha} d_{r}} F_{\alpha} E_{2}\right. \\
& \left.+\frac{\left(\left(w+v_{0 \alpha}\right)^{2}-\mathrm{v}_{0 i}^{2} t_{c}+\mathrm{y}^{2} / 2\right)}{v_{T \alpha} \omega_{c \alpha} k_{0} R} \frac{\partial F_{\alpha}}{u \partial u} E_{1} \sin \vartheta-\frac{w E_{3}}{k_{0} v_{T \alpha}^{2}} \frac{\partial F_{\alpha}}{u \partial u}\right] .
\end{aligned}
$$

Here, $E_{1,2,3}$ are the radial, bi-normal, and parallel components of the wave field; $\Omega=\omega R_{0} q / \nu_{T \alpha}$ is the normalized wave frequency; $\omega_{c \alpha}=e_{\alpha} B / m_{\alpha} c$ are the cyclotron frequencies; $k_{0}=h_{\vartheta} / r=1 / q R$ is the parallel wave vector; $h_{\vartheta}$ $=B_{\vartheta} / B_{0}$ is the magnetic field inclination; $w=v_{\|} / v_{T \alpha}$, $y=v_{\perp} / v_{T \alpha}$, and $u=\sqrt{w^{2}+y^{2}}$ are the normalized space velocities; $\partial n_{0} / \partial r=-n_{0} / d_{r}$ is the density gradient; $\eta_{e, i}=\partial \ln T_{e, i} / \partial \ln n_{0}$; and $v_{T \alpha}=\sqrt{T_{\alpha} / m_{\alpha}}$ is the particle thermal velocities. It is assumed that unbalanced NB injection may drive ion rotation with shifted Maxwell distribution $F_{\alpha}=\left.F_{M \alpha}\right|_{w=w-v 0 \alpha}$ with $v_{0 \alpha}=V_{0 \alpha} / v_{T \alpha}$ having the respective parallel equilibrium velocity $V_{0 i}$, accounting for the centrifugal effect with the parameter $t_{c} \approx T_{e} /\left(T_{e}+T_{i}\right)$ for ions. ${ }^{24}$ Due to the ohmic current, a shifted distribution is also valid for electrons. To model the respective equilibrium distribution for the energetic ions, we take into account the dynamic friction of NB ions with electrons, considering $V_{\mathrm{h}} \ll V_{\mathrm{Te}}$, and strong scattering by ions below critical velocity, ${ }^{20}$ i.e., $V_{\mathrm{h}}<V_{\text {cr. }}$. Within this model, the energetic particles can be described by a "bump-on-tail like" distribution, $F_{h}$ $=C v^{2} g(\lambda) \exp \left[-v^{2} / 2 v_{T h}^{2}\right]$, with hot ion temperature $T_{\mathrm{h}}$ $\approx \mathrm{O}\left(m_{\mathrm{b}} V_{\mathrm{cr}}^{2} / 6\right)$ and Gaussian angular distribution (similar to Refs. 15 and 17) $g(\lambda)=\frac{1}{\Delta \sqrt{\pi}} \exp \left[-\left(\lambda-\lambda_{0}\right)^{2} / \Delta^{2}\right], \lambda_{0}=\frac{v_{\perp b}^{2}}{v_{b}^{2}}$, with maximum at the injection pitch angle $\frac{v_{\| b}}{v_{b}}=\sqrt{\left(1-\lambda_{0}\right)}$, $C=\frac{n_{\mathrm{h}} \sqrt{\left(1-\lambda_{0}\right)}}{6\left(2 \pi \mathrm{v}_{\mathrm{Th}}^{2}\right)^{3 / 2}}$ is the normalization coefficient, and $\Delta \propto T_{h}$ is some characteristic of the angular thermal spread.

We expect that the GAM frequency be of the order of the critical circulation one and much higher than the ion transit frequency, i.e., $\omega_{G A M} \sim V_{\text {cr }} / R q \gg v_{T i} / R q$, which leads to fluid results for the main plasma particles. ${ }^{14,24}$ Keeping the leading terms in the respective electron and ion radial current perturbations driven by the radial magnetic drift velocity $V_{r \alpha}=-\left[\left(w-v_{0 i}\right)^{2}-v_{0 i}^{2} \tau_{e} /\left(1+\tau_{e}\right)+y^{2} / 2\right] v_{T \alpha}^{2} \sin \vartheta / R \omega_{c \alpha}$, we get the expressions for the average radial components of the perturbed electron and ion current densities, respectively,

$$
\begin{aligned}
\left\langle\tilde{j}_{r}^{e}\right\rangle= & \frac{e_{i}^{2} q n_{0 e}}{m_{i} \omega_{c i}}\left[\left(1+\frac{v_{0 i}^{2}}{2}\left(1-\tau_{e}\right)\right) E_{c}\right. \\
& \left.-\frac{\sqrt{2 \pi}}{8}\left(2 v_{0 e}+\tau_{e} \Omega_{i}^{*}\left(2+\eta_{i}\right)\right) E_{s}\right],
\end{aligned}
$$

$$
\begin{aligned}
\left\langle\dot{j}_{r}\right\rangle= & \frac{e_{i}^{2} n_{0 i} q}{m_{i} \omega_{c i} \Omega}\left\{\left[\frac{2}{\Omega}-\mathrm{i} \frac{\sqrt{2 \pi}}{4} \Omega^{2}\left(\Omega^{2}+1\right) \exp \left(-\frac{\Omega^{2}}{2}\right)\right] E_{c}\right. \\
& -\mathrm{i}\left(\Omega_{i}^{*}\left(1+\eta_{i}\right)+v_{0 i}\right) E_{s}+\frac{v_{T i}}{R_{0} \omega_{c i}} \\
& \times\left[\mathrm{i}\left(\frac{7}{2}+\frac{23}{2 \Omega^{2}}+2 v_{0 i}^{2}\left(2-t_{c}\right)\right)\right. \\
& \left.\left.+\sqrt{2 \pi} \frac{\Omega}{4}\left(\Omega^{4}+2 \Omega^{2}+2\right) \exp \left(-\frac{\Omega^{2}}{2}\right)\right] E_{1}\right\},
\end{aligned}
$$

where $\Omega_{i}^{*}=v_{T i} / d_{r} \omega_{c i} h_{p}$ and $\Omega=\omega R_{0} q / v_{T i}$ are the normalized drift and wave frequencies, respectively, $\tau_{e}=T_{e} / T_{i}$, and the term $\left.v_{0 i}^{2} \tau_{e} /\left(1+\tau_{e}\right)\right) v_{T \alpha}^{2}$ is responsible for centrifugal correction of the magnetic drift via the poloidal variation of electrostatic potential over the magnetic surface. Then, using the quasi-neutrality condition for the electron and ion densities, ${ }^{14,24}$ we find the electric field amplitudes $E_{S, c}$ to complete the calculations of the radial currents

$$
\begin{gathered}
E_{s} \approx \\
\frac{v_{T i} \tau_{e} E_{1}}{R_{0} \omega_{c i}}\left(\frac{2}{\Omega^{2}}\left(\tau_{e} \Omega_{i}^{*}\left(1+\eta_{i}\right)+2 v_{0 i}\right)\right. \\
\left.+\mathrm{i} \sqrt{2 \pi} \frac{v_{0 e}}{\Omega}-\sigma \frac{n_{\mathrm{h}} v_{T i}}{\Omega^{2} v_{T h}} N_{\mathrm{c}}\right), \\
E_{c}=\frac{\tau_{e} v_{T i}}{R_{0} \omega_{c i} \Omega}\left\{\mathrm{i}\left[2+v_{0 i}^{2}\left(1-t_{c}\right)\right]-\mathrm{i} n_{h} \frac{v_{T i}}{v_{T h}} N_{\mathrm{s}}\right. \\
\left.-\sqrt{2 \pi}\left[2 \frac{v_{0 e}}{\Omega}\left(v_{0 i}+\Omega_{i}^{*}\right)-\frac{\Omega^{3}}{2} \exp \left(-\frac{\Omega^{2}}{2}\right)\right]\right\} E_{1} .
\end{gathered}
$$

Here, the density perturbation response via the coefficients, $N_{\mathrm{c}, \mathrm{s}} \approx O(1)$, driven by the energetic ions is assumed to be small because it is proportional to the relative density of energetic ions $n_{h}=n_{0 h} / n_{0}$ and $v_{T i} \ll v_{T h}$.

To evaluate the effect of energetic ions in Eq. (1), we follow the standard procedure by taking the first terms, $E_{3}=E_{s} \sin \vartheta+E_{c} \cos \vartheta$ and $f_{h}=f_{s, h} \sin \vartheta+f_{c, h} \cos \vartheta$, of Fourier expansion of the respective function. Equilibrating the $\sin \vartheta / \cos \vartheta$ coefficients after substitution of the $E_{3}$-field and respective distribution function into Eq. (1) and ignoring the hot species rotation, drift, and second poloidal harmonic effects, we obtain equations for the perturbed distributions ${ }^{14}$

$$
\begin{aligned}
f_{s, h}= & \frac{\mathrm{i} e_{h} \Omega_{h} q\left(\mathrm{w}^{2}+\mathrm{y}^{2} / 2\right) E_{1}}{\omega_{c h} m_{h} v_{T h}\left(\Omega_{h}^{2}-\mathrm{w}^{2}\right)} \frac{\partial F_{h}}{u \partial u} \\
& -w \frac{e_{\alpha} q R\left(\mathrm{i} \Omega_{h} E_{s}-w E_{c}\right)}{T_{h}\left(\Omega_{h}^{2}-\mathrm{w}^{2}\right)} \frac{\partial F_{h}}{u \partial u}, \\
f_{c, h}= & \frac{e_{h} q w\left(w^{2}+y^{2} / 2\right) E_{1}}{\omega_{c h} m_{h} v_{T h}\left(\Omega^{2}-\mathrm{w}^{2}\right)} \frac{\partial F_{h}}{u \partial u} \\
& -w \frac{e_{h} q R\left(\mathrm{i} \Omega_{c}+w E_{s}\right)}{T_{h}\left(\Omega^{2}-\mathrm{w}^{2}\right)} \frac{\partial F_{h}}{u \partial u} .
\end{aligned}
$$

Here, the bump rotation $v_{0 h}=0$ is omitted because it is included into the basic ion rotation, the finite orbit width effect $^{25}$ is not included due to the proposed geodesic continuum study, and the diamagnetic effect ${ }^{24}$ is ignored in the energetic ion distribution, consistently with the assumption of plasma ion pressure larger than the hot ion pressure. In 
fact, it is rather difficult to carry out analytical calculations taking into account these effects; therefore, this will be the subject of a later separate study. Next, using the sin-component distribution from Eq. (6a), we have to calculate the $\vartheta$ averaged radial current

$$
\left\langle\hat{j}_{r}^{h}\right\rangle=e_{h} v_{T h}^{3} \int_{0}^{\infty} u^{2} d u \oint d \vartheta \sum_{\sigma= \pm 1} \int_{0}^{1} \frac{V_{r h} f_{s, h} d \lambda}{2 \sqrt{1-\lambda}}
$$

which is driven by hot ions. Changing variables $y=\sqrt{\lambda} u$ and $w=\sigma \sqrt{(1-\lambda)} u$ in Eq. (6a) where $\sigma= \pm 1$ for co- and counter injection, respectively, we may easily proceed with integration over the $u$-velocity using standard incomplete Gamma and $Z(x)$-plasma dispersion functions. To demonstrate the evaluation procedure of an unwieldy equation, we show the intermediate results of integration over $u=0, \infty$ related to the current driven by the electric field. Below, the $E_{S}$-component of the current is presented separately from others

$$
\begin{aligned}
\left\langle\dot{j}_{r s}^{h}\right\rangle= & \mathrm{i} \sigma \frac{\sqrt{2} j_{0} n_{h}}{12 E_{1}} \frac{R \omega_{c h} E_{s}}{v_{T h} \pi} \int_{0}^{1} \sqrt{1-\lambda_{0}} \frac{d \lambda}{\Delta} \exp \left[-\frac{\left(\lambda-\lambda_{0}\right)^{2}}{\Delta^{2}}\right] \\
& \times \frac{(2-\lambda)}{(1-\lambda)^{2}} \Omega_{h}\left\{\frac{\Omega_{h}^{4}}{(1-\lambda)^{2}}+4-4 \frac{\left(\lambda-\lambda_{0}\right) \lambda}{\Delta^{2}}\right. \\
& \times\left(\frac{\Omega_{h}^{2}}{(1-\lambda)}+2\right)+\frac{\Omega_{h}^{4}}{(1-\lambda)^{2}}\left[\left(\frac{\Omega_{h}^{2}}{2(1-\lambda)}-1\right.\right. \\
& \left.\left.-2 \frac{\left(\lambda-\lambda_{0}\right) \lambda}{\Delta^{2}}\right)\right] \times\left[\operatorname{Re}\left(\Gamma\left(0, \frac{-\Omega_{h}^{2}}{2(1-\lambda)}\right)\right)+\mathrm{i} \pi\right] \\
& \left.\times \exp \left(-\frac{\Omega_{h}^{2}}{2(1-\lambda)}\right)\right\}
\end{aligned}
$$

where $j_{0}=\frac{e_{h}^{2} n_{0} q v_{T h}}{m_{h} R_{0} \omega_{c h}^{2}} E_{1}$ and the integration $\int_{0}^{\infty} \frac{d t^{2}}{2} \frac{\exp \left(-t^{2} / 2\right)}{\left(t^{2}-x^{2}\right)}$ $=\frac{1}{2} \exp \left(-\frac{x^{2}}{2}\right)\left[\operatorname{Re}\left(\Gamma\left(0,-\frac{x^{2}}{2}\right)\right)+\mathrm{i} \pi\right]$ is completed using the real part of $\Gamma\left(0,-x^{2} / 2\right)=\exp \left(-x^{2} / 2\right) \int_{-x^{2} / 2}^{\infty} d z \exp (-z) / 2 z$, that is, the incomplete Gamma function, which is equivalent to the $E_{1}\left(-x^{2} / 2\right)$ function, ${ }^{26}$ where $x=\Omega_{h} / \sqrt{(1-\lambda)}$. The rest of the current is

$$
\begin{aligned}
\left\langle\hat{j}_{r r, c}^{h h}\right\rangle= & \frac{j_{0} n_{h}}{12 \sqrt{\pi}} \int_{0}^{1} \frac{d \lambda}{\Delta} \exp \left[-\frac{\left(\lambda-\lambda_{0}\right)^{2}}{\Delta^{2}}\right] \frac{\sqrt{1-\lambda_{0}}}{\sqrt{1-\lambda}} \frac{(2-\lambda)}{(1-\lambda)^{2}} \\
& \times\left[\frac{R \omega_{c h}}{v_{T h}} E_{c}+\mathrm{i} \frac{(2-\lambda) \Omega_{h}}{2(1-\lambda)} E_{1}\right] \\
& \times\left\{\left[\frac{-\Omega_{h}^{6}}{(1-\lambda)}+\Omega_{h}^{4}-\Omega_{h}^{2}(1-\lambda)-9(1-\lambda)^{2}\right.\right. \\
& \left.+4\left(\Omega_{h}^{4}+\Omega_{h}^{2}(1-\lambda)+3(1-\lambda)^{2}\right) \frac{\left(\lambda-\lambda_{0}\right) \lambda}{\Delta^{2}}\right] \\
& -\frac{\sqrt{2} \Omega_{h}^{5}}{2 \sqrt{(1-\lambda)}}\left(\frac{\Omega_{h}^{2}}{(1-\lambda)}-2-4 \frac{\left(\lambda-\lambda_{0}\right) \lambda}{\Delta^{2}}\right) \\
& \left.\times Z\left(\frac{\Omega_{h}}{\sqrt{2(1-\lambda)}}\right)\right\}
\end{aligned}
$$

where $\quad$ integral $^{26} \quad \frac{1}{\sqrt{\pi}} \int_{0}^{\infty} d t \frac{\exp \left(-t^{2} / 2\right)}{\left(t^{2}-x^{2}\right)}=\frac{\sqrt{\pi}}{2 x} \exp \left(-x^{2} / 2\right)(\mathrm{i}$ $-\operatorname{erfi}(x / \sqrt{2}))=\frac{1}{2 x} Z(x / \sqrt{2})$ is presented by the plasma dispersion function $Z(\hat{x})=\frac{1}{\sqrt{\pi}} \int_{-\infty}^{\infty} d t \frac{\exp \left(-t^{2}\right)}{(t-\hat{x})}$ with $\hat{x}=\frac{\Omega_{h}}{\sqrt{2(1-\lambda)}}$. To further proceed with the evaluation of Eqs. (7a) and (7b), the Laplace method of integration over $\lambda$ may be used in the asymptotic limit $\lambda_{0}^{2},\left(1-\lambda_{0}\right)^{2} \gg \Delta^{2}$ for the integrals same as in Eqs. (7a) and (7b)

$$
\begin{aligned}
I\left(\lambda_{0}\right)= & \int_{0}^{1} \frac{d \lambda}{\sqrt{\pi} \Delta}\left[\Phi_{0}(\lambda)+\frac{\left(\lambda-\lambda_{0}\right)}{\Delta^{2}} \Phi_{1}(\lambda)\right] \\
& \times \exp \left[-\frac{\left(\lambda-\lambda_{0}\right)^{2}}{\Delta^{2}}\right] \approx \frac{1}{2}\left[\left(\Phi\left(\lambda_{0}\right)+\frac{\Phi^{\prime}{ }_{1}\left(\lambda_{0}\right)}{2}\right.\right. \\
& \left.+\frac{\Delta^{2}}{4} \Phi^{\prime \prime}\left(\lambda_{0}\right)+O\left(\Delta^{4}\right)\right) \operatorname{erf}\left(\frac{\left(\lambda-\lambda_{0}\right)}{\Delta}\right) \\
& \left.+O\left(\Phi_{0,1}\right) \exp \left(-\frac{\left(\lambda-\lambda_{0}\right)^{2}}{\Delta^{2}}\right)\right]_{0}^{1}
\end{aligned}
$$

where $\Phi_{0,1}(\lambda)$ are some arbitrary functions, whose derivatives are assumed to be not large, $\operatorname{erf}(x)=2 \int_{0}^{x} \exp \left(-z^{2}\right) d z$ / $\sqrt{\pi}$ is the probability integral ${ }^{26}$ that has asymptotic properties, $\operatorname{erf}\left(\left(1-\lambda_{0}\right) / \Delta\right) \approx 1$, and $\operatorname{erf}\left(-\lambda_{0} / \Delta\right) \approx-1$. The above integration method is applied to Eqs. (7a) and (7b), and omitting the $\Delta^{2}$-corrections, we obtain

$$
\begin{aligned}
\left\langle\hat{j}_{r, s}^{h}\right\rangle= & \mathrm{i} \sigma \frac{\sqrt{2}}{12} \frac{j_{0} n_{h}}{\sqrt{\pi} E_{1}} \frac{R \omega_{c h} \Omega_{h} E_{s}}{v_{T h}\left(1-\lambda_{0}\right)^{3 / 2}}\left[\frac{\left(2-\lambda_{0}\right) \Omega_{h}^{4}}{\left(1-\lambda_{0}\right)^{2}}\right. \\
& -4 \frac{\Omega_{h}^{2}}{\left(1-\lambda_{0}\right)}-4 \lambda_{0}+\Omega_{h}^{4}\left(\frac{\left(2-\lambda_{0}\right) \Omega_{h}^{2}}{2\left(1-\lambda_{0}\right)^{3}}-\frac{\left(4-\lambda_{0}\right)}{\left(1-\lambda_{0}\right)^{2}}\right) \\
& \left.\times\left[\operatorname{Re}\left(\Gamma\left(0, \frac{-\Omega_{h}^{2}}{2\left(1-\lambda_{0}\right)}\right)\right)+\mathrm{i} \pi\right] \exp \left(\frac{-\Omega_{h}^{2}}{2\left(1-\lambda_{0}\right)}\right)\right],
\end{aligned}
$$

$$
\begin{aligned}
\left\langle j_{r, r c}^{h h}\right\rangle= & \frac{j_{0} n_{h}}{12 E_{1}}\left\{\frac { R \omega _ { c h } E _ { c } } { v _ { T h } ( 1 - \lambda _ { 0 } ) } \left[\frac{\left(\lambda_{0}-2\right) \Omega_{h}^{6}}{\left(1-\lambda_{0}\right)^{3}}+\frac{\left(6-\lambda_{0}\right) \Omega_{h}^{4}}{\left(1-\lambda_{0}\right)^{2}}\right.\right. \\
& +\frac{\left(2+\lambda_{0}\right) \Omega_{h}^{2}}{\left(1-\lambda_{0}\right)}-3\left(2-3 \lambda_{0}\right)+\frac{\sqrt{2} \Omega_{h}^{5}}{2\left(1-\lambda_{0}\right)^{5 / 2}} \\
& \left.\times\left(8-2 \lambda_{0}-\frac{\left(2-\lambda_{0}\right) \Omega_{h}^{2}}{\left(1-\lambda_{0}\right)}\right) Z\left(\frac{\Omega_{h}}{\sqrt{2\left(1-\lambda_{0}\right)}}\right)\right] \\
& +\mathrm{i} \frac{\left(2-\lambda_{0}\right) \Omega_{h}}{2\left(1-\lambda_{0}\right)^{2}} E_{1}\left[\frac{\left(\lambda_{0}-2\right) \Omega_{h}^{6}}{\left(1-\lambda_{0}\right)^{3}} \frac{\left(6+\lambda_{0}\right) \Omega_{h}^{4}}{\left(1-\lambda_{0}\right)^{2}}\right. \\
& +\frac{\left(2+3 \lambda_{0}\right) \Omega_{h}^{2}}{\left(1-\lambda_{0}\right)}-6+15 \lambda_{0}-\frac{\sqrt{2} \Omega_{h}^{5}}{2\left(1-\lambda_{0}\right)^{5 / 2}} \\
& \left.\left.\times\left(\frac{\left(2-\lambda_{0}\right) \Omega_{h}^{2}}{\left(1-\lambda_{0}\right)}-8\right) Z\left(\frac{\Omega_{h}}{\sqrt{2\left(1-\lambda_{0}\right)}}\right)\right]\right\}
\end{aligned}
$$

where $\sigma=0$ for balanced NB. It should be noted that the above result is not dependent on the $\Delta$-width in the limit $\lambda_{0}^{2},\left(1-\lambda_{0}\right)^{2} \gg \Delta^{2}$. 
Furthermore, taking into account the electric field amplitudes $E_{s, c}$ from Eq. (5) in the sum of the components of current species $J_{\Sigma}=\left\langle\tilde{j}_{r}^{i}+\tilde{j}_{r}^{i}+\tilde{j}_{r}^{h}\right\rangle+j_{p}$ together with the ion radial polarization current, $j_{p}=-\mathrm{i} \omega c^{2} E_{1} / 4 \pi c_{A}^{2}$, where $c_{A}=B / \sqrt{4 \pi n_{i} m_{i}}$, we obtain the total radial current valid for the GAM dispersion $\left(\operatorname{Im} J_{\Sigma} E_{1}^{*}=0\right)$ and instability $\left(\operatorname{Re} J_{\Sigma} E_{1}^{*}<0\right)$ analyses

$$
\begin{aligned}
J_{\Sigma}= & \frac{e_{i}^{2} n_{0 i} q v_{T h}}{m_{i} \omega_{i}^{2} R}\left\{\left\langle i_{r}^{h}\right\rangle / j_{0}+\frac{\mathrm{i}}{\Omega} \frac{v_{T i}}{v_{T h}}\left(\frac{7}{2}+2 \tau_{e}+4 v_{0 i}^{2}-\frac{\Omega^{2}}{q^{2}}\right)\right. \\
& +\sqrt{2 \pi} \frac{v_{T i}}{v_{T h}}\left[\frac{\Omega^{2}}{4}\left(\Omega^{2}+4 \tau_{e}+2\right) \exp \left(-\frac{\Omega^{2}}{2}\right)\right. \\
& \left.\left.-2 \frac{v_{0 e}^{*} v_{0 i}^{*}}{\Omega^{2}}\right]\right\} E_{1},
\end{aligned}
$$

where the toroidal rotation term $v_{0 i}$ is extended to the earlier calculated diamagnetic effect ${ }^{14,24} v_{0 i}^{*}=v_{0 i}+\left(1+\eta_{i}+3 \tau_{e} /\right.$ 2) $\Omega_{i}^{*} / 2, v_{0 e}^{*} \approx v_{0 e}$.

Generally, the evolution of the wave amplitude is determined by the condition that the time rate of change in wave energy $W$ is equal to the negative of the power dissipated into the background plasma plus the power of the energetic particles transferred to the waves, which is the integral of the power density over the coordinate space $\partial W / \partial t=-\int \operatorname{Re} J_{\Sigma} E_{1}^{*} d^{3} r$. Here, we study the wave stability in the local continuum approximation that leads to the resonance condition $J_{\Sigma}=0$; the instability growth rate can be calculated perturbatively by assuming that the imaginary part of the frequency $\omega=\omega_{G A M}+i \gamma$ is small in comparison to the real part $\gamma \ll \omega_{G A M}$. Using Eq. (9) in the implicit form of $\omega$ after multiplication by the $E_{1}^{*}$-complex conjugate part of $E_{1}$ to eliminate the time oscillation factor and expanding the equation $J_{\Sigma} E_{1}^{*}=0$ in Taylor series of $\gamma$, we have the resonance condition $\operatorname{Im} J_{\Sigma} E_{1}^{*}=0$ and $\partial \operatorname{Im} J_{\Sigma} E_{1}^{*} /$ $\left.\partial \omega\right|_{\operatorname{Im} J_{\Sigma} E^{*}=0} \gamma=\operatorname{Re} J_{\Sigma} E_{1}^{*}$. It is evident that the GAM stability analysis using Eq. (8) is rather involved; therefore, we initially circumvent this difficulty by finding the region where the instability may occur ignoring the exact GAM dispersion. In the case of a local approximation, we may use the power density $P=$ $J_{\Sigma} E_{1}^{*}$ to define the instability region. Then, the correct GAM dispersion is found in the instability region. We also assume that the additional dissipated power related to the parallel electric field, Eqs. (4) and (5), is small in Eq. (8), i.e., proportional to $T_{e} / T_{h} \approx 2 / 15$, and it may be ignored $\left(E_{c, s}=0\right)$ in comparison to the $E_{1}$-component effect for a preliminary investigation.

\section{NUMERICAL RESULTS AND DISCUSSION}

We begin analyzing the possible region for the GAM instability using the normalized dissipated/emitted power density $P_{\Sigma}=\operatorname{Re}\left\langle\tilde{j}_{\Sigma} E_{1}^{*}\right\rangle /\left(n_{h} j_{0} E_{1}^{*}\right)$. It is plotted as a function of the normalized frequency $\Omega_{h}$ and $\lambda_{0}$ in Fig. 1, where negative power means instability drive. The maximum of power emission

$$
P_{\max }=\frac{125 \sqrt{2 \pi}}{2^{4}\left(1-\lambda_{0}\right)^{3 / 2}\left(2-\lambda_{0}\right)^{2}} \exp \left(\frac{-5}{2\left(2-\lambda_{0}\right)}\right)
$$

is found at the frequency $\Omega_{\max } \approx \sqrt{5\left(1-\lambda_{0}\right) /\left(2-\lambda_{0}\right)}$ and the region of instability is upper bounded by the condition

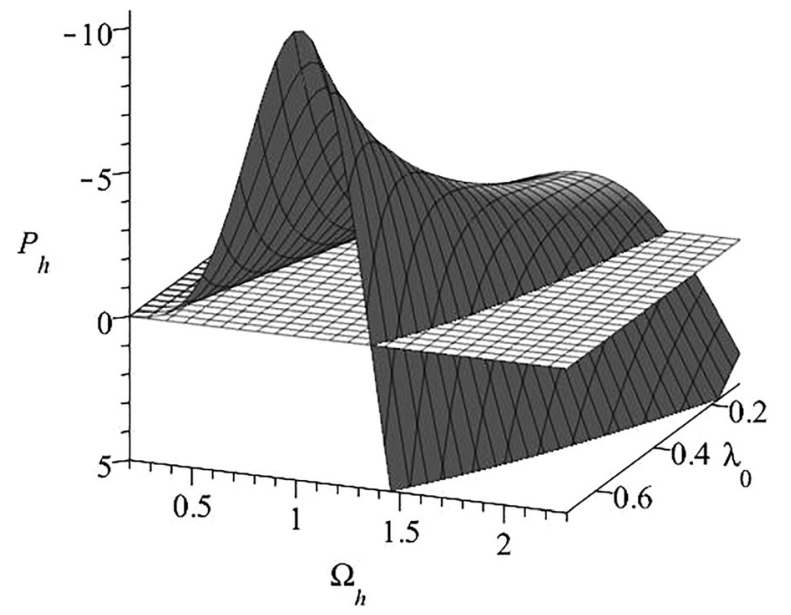

FIG. 1. Plot of normalized emitted power density driven by energetic ions as a function of the normalized frequency $\Omega_{h}$ and $\lambda_{0}$-value for $n_{h}=5 \%$, $\tau_{e}=1.5, T_{h} / T_{e} \approx 15 / 2$, and $E_{c, s}=0$.

$\Omega_{h}<\Omega_{\text {ulim }}=2 \sqrt{2\left(1-\lambda_{0}\right) /\left(2-\lambda_{0}\right)}$, due to the strong Landau damping in the hot ions which has the maximum of the order of $O\left(P_{\max }\right)$ at

$$
\Omega_{\max 2} \approx 2 \sqrt{\left(3-\lambda_{0}+\sqrt{3}-\sqrt{3} \lambda_{0} / 2\right)\left(1-\lambda_{0}\right) /\left(2-\lambda_{0}\right)},
$$

which is partly shown in Fig. 1 and the Landau damping in the hot ions begins to be exponentially small for $\Omega_{h}>1.5 \Omega_{\max 2}$, where the geodesic modes may be indirectly excited due to the modification of equilibrium parameters such as the diamagnetic drift or rotation. ${ }^{14,24}$

The criterion for the geodesic mode instability $\operatorname{Re} J_{\Sigma} E_{1}^{*}<0$ is used in the form

$$
\begin{aligned}
-n_{h} P_{h}>P_{\text {Landau }}= & \frac{\sqrt{2 \pi}}{4} \frac{v_{T h}}{v_{T i}} \Omega_{h}^{2}\left(\frac{v_{T h}^{2}}{v_{T i}^{2}} \Omega_{h}^{2}+4 \tau_{e}+2\right) \\
& \times \exp \left(-\frac{\Omega_{h}^{2}}{2} \frac{v_{T h}^{2}}{v_{T i}^{2}}\right) .
\end{aligned}
$$

Assuming that all ions are identical and choosing the critical energy at the energetic ion distribution maximum and $T_{h} / T_{e} \approx 15 / 2$, both parts of Eq. (11) are plotted as a function of $\Omega_{\mathrm{h}}$ for $n_{h}=5 \%, \lambda_{0}=0.3,0.7$, and $\tau_{e}=1,1.5$ in Fig. 2. The instability may appear in the frequency region, where dash and/or dash-dotted lines related to the instability drive power density $\left(-n_{h} P_{h}\right)$ stay above solid or dashed lines, which mark the $P_{\text {Landau }}$-power densities responsible for the ion Landau damping. Here, the driving power density may be shifted up or down for another energetic ion density in the respective relation to the calculated value $n_{h}$ and the respective instability may appear when the $n_{h}$-value stays above some threshold value $n_{\mathrm{th}}$.

To clarify the lower bound frequency, the GAM instability threshold $n_{\mathrm{th}}$, which is defined by Landau damping on basic and hot ions, is calculated for $\lambda_{0}=0.6$ and different electron temperatures $\tau_{e}=0.8,1,1.2$, and 1.5. In Fig. 3, we see that the instability region $n_{h} \geq n_{t h}$ begins to be strongly lower bounded with diminishing of the electron temperature 


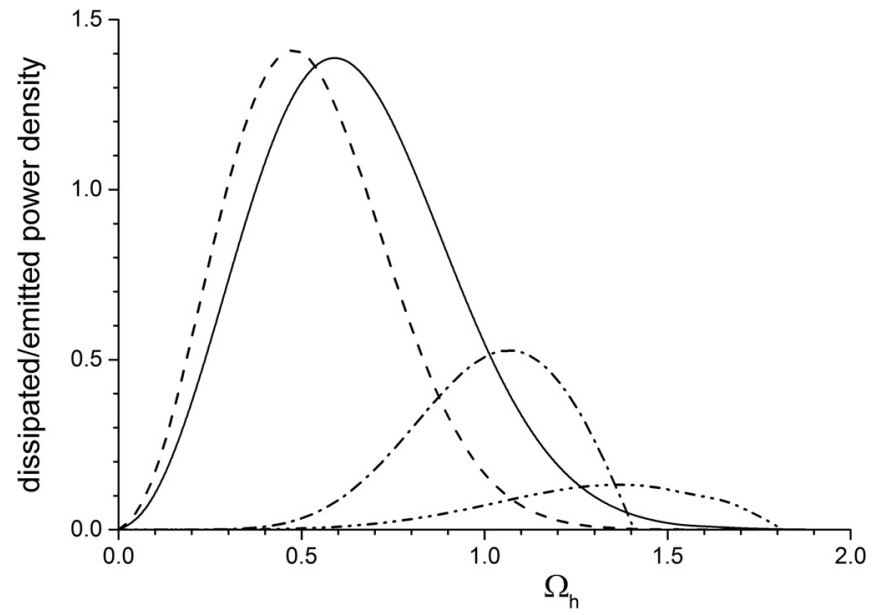

FIG. 2. Plot of normalized dissipated power due to Landau damping in plasma ions ( $\tau_{e}=1$-solid and $\tau_{e}=1.5$-dashed lines) and driving power by energetic ions for $n_{h}=5 \%, \lambda_{0}=0.3,0.7$ represented as dash-dot-dotted and dash-dotted lines, respectively.

$\tau_{e}<1$. For higher electron temperatures, the Landau dumping begins to be very small and the GAM instability may even occur with a very small concentration of the energetic ions. In this case, $\lambda_{0} \approx 0.5$ and $\tau_{e}=1.2$, we obtain the instability condition criteria $n_{h}>3 \%$ at the frequency $\Omega_{\mathrm{h}}$ $\approx 0.92 \Omega_{\max }=1.16$ of the power density emission maximum in Fig. 1 and the GAM instability is approximately limited by the frequency band $0.92 \Omega_{\max }<\Omega_{h}<\Omega_{\text {ulim. }}$. Next, the GAM dispersion is analyzed using the resonance condition $\operatorname{Im} J_{\Sigma}=0$ in Eq. (9), with respect to the instability limitations shown in Figs. 1-3. The developed theory is valid in the limit of large values of the safety factor, $q^{2} \gg 1$, but the GAM instability spectrum may only appear for $q \leq 3.5$, due to the upper bounded frequency limitation.

We begin the GAM dispersion study in the unstable region for $q=2.8$ and $n_{h}=3 \%$. The results are plotted in Fig. 4 as a function of the pitch angle for $\tau_{e}=1,1.4$, and 2. Three geodesic modes appear for $\lambda_{0}>0.4$ : the lowest frequency mode is unstable, the intermediate mode is strongly dissipated in hot ions, and the highest mode is slightly

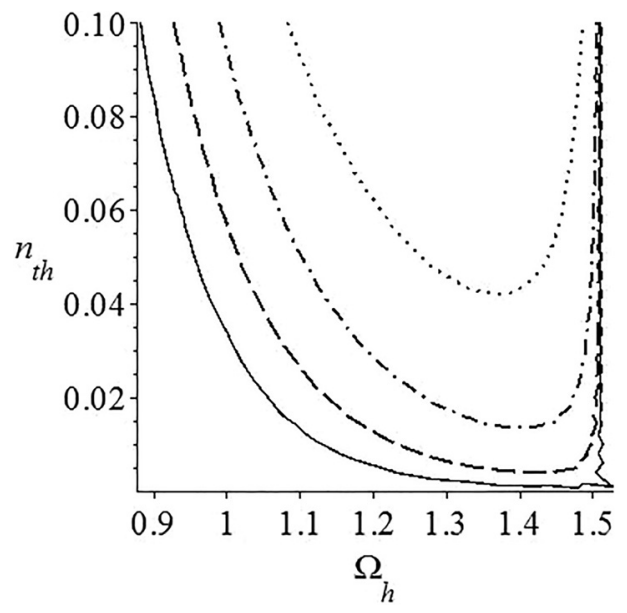

FIG. 3. Plot of the geodesic instability threshold $n_{t h}$ as a function of $\Omega_{\mathrm{h}}$ for $\lambda_{0}=0.6$ and different electron temperatures $\tau_{e}=0.8,1,1.2$, and 1.4 (dot, dash-dotted, dashed, and solid lines, respectively).

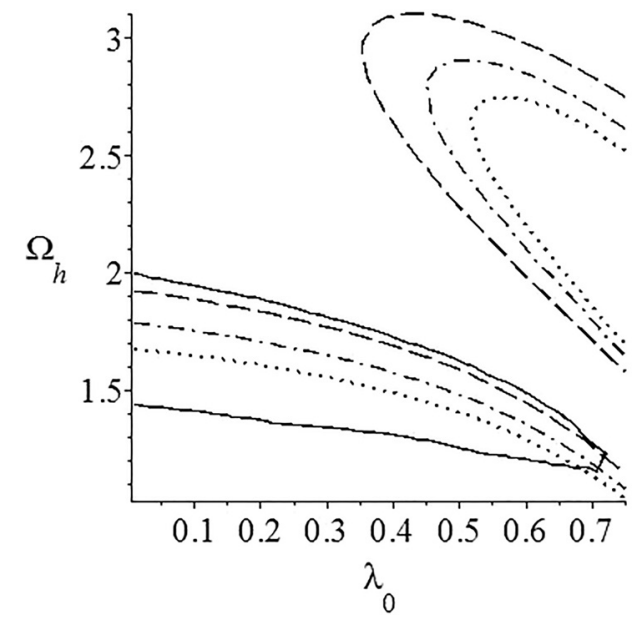

FIG. 4. Plot of normalized geodesic frequency $\Omega_{h}=\omega R_{0} q / v_{T h}$ as a function of $\lambda_{0}$ for $n_{h}=3 \%, q=2.8$, and $\tau_{e}=1,1.4,2$ (dash, dash-dotted and dotted lines, respectively). Solid line $\left(\tau_{e}=1\right.$ and $\left.n_{h}=3 \%\right)$ shows the frequency limit for the GAM instability region, demonstrating that all lower frequency modes are unstable.

dissipated. Here, the GAM spectrum for $q=2.8$ is plotted slightly below the instability threshold and the curves move down to the instability region for lower $q$-values. However, the instability region stays below the maximum of the circulation frequency at the bump maximum, and it is limited by the Landau damping in the hot ions $\Omega_{h}<2 \sqrt{2\left(1-\lambda_{0}\right) /\left(2-\lambda_{0}\right)}$, which is similar to limitations discussed in Refs. 17 and 27. The lower bounded condition is defined by competition between the ion Landau damping in Eq. (9) and the driving power by energetic ions in Eq. (8), shown in Figs. 1-3.

It should be noted that the substantial deviation of the unstable region may appear for $\Delta \geq 0.3$ near the trapped hot ion region $\lambda_{0} \approx 1-\varepsilon$, where the bounce effect may be strong and has to be taken into account; however, the unstable GAM/EGAM frequencies stay far from bounce resonances $\Omega_{h} \gg \sqrt{r / R_{0}}$. To calculate the effect analytically, a new technique has to be developed, and the results will be published elsewhere.

\section{A. Discussion of the GAM-EGAM difference}

To understand the behavior of the geodesic mode dispersion splitting on the standard GAM and EGAM, we plot the geodesic frequency as a function of the energetic ion concentration in Fig. 5, for $\lambda_{0}=0.6, q=2.8$, and different electron temperatures $\tau_{e}=1,1.4,1.6$, and 2 . For a small energetic ion concentration and low electron temperature $\tau_{e} \leq 1.6$, the normalized GAM frequency grows with $n_{h}$, as approximately described by formula $\Omega_{h, G}^{2} \approx q^{2}\left[\frac{1}{15}\left(4+\frac{7}{\tau_{e}}\right)+n_{h} \Lambda\left(\lambda_{0}\right)\right]$, where $\Lambda\left(\lambda_{0}\right) \approx \frac{5}{8} \frac{\left(2-\lambda_{0}\right)\left(6-7 \lambda_{0}\right)}{\left(1-\lambda_{0}\right)}$ for $\Omega_{h, G}^{2} \gg 1$, with the respective dependence shown by dotted and dash-dotted lines in Fig. 5.

This GAM branch has exponentially small Landau damping for $n_{h} v_{T h}^{2} / v_{T i}^{2} \ll 1$, which is also confirmed by Eq. (11). Above some density threshold of the order of $n_{h, \text { cr }} \approx 1-2 \%$, two EGAM branches appear. ${ }^{15,18,27}$ The higher mode with asymptotic frequency $\Omega_{1, E G A M} \approx \Omega_{\max 2}$ is strongly 


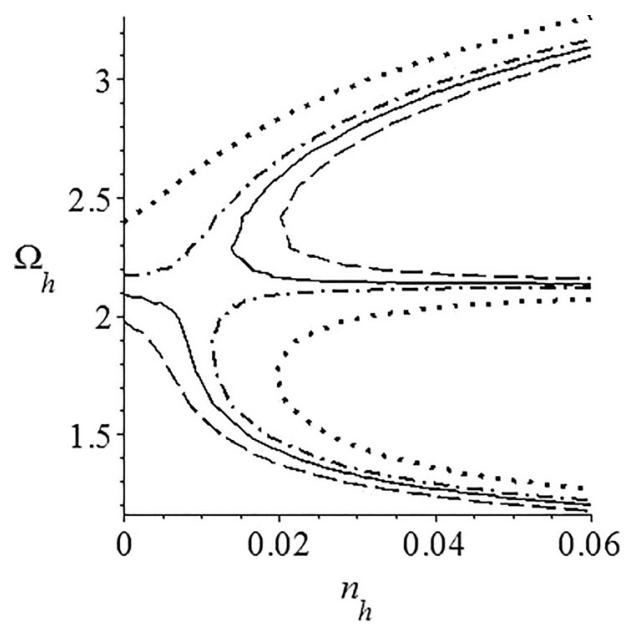

FIG. 5. Plot of the geodesic frequencies as a function of the hot ion density $n_{h}$ for $\lambda_{0}=0.6, q=2.8$, and different electron temperatures $\tau_{e}=1,1.4,1.6$, and 2 (dotted, dash-dotted, solid, and dashed lines, respectively).

dissipative, and the lower EGAM branch is unstable in the region $\Omega_{h}<\Omega_{\text {uplim }}=2 \sqrt{2\left(1-\lambda_{0}\right) /\left(2-\lambda_{0}\right)}$ together with the threshold shown in Fig. 3. For quasi parallel injection $\lambda_{0} \approx 0$, the upper limit coincides with critical (or maximum) circulation frequency $\omega_{G}<V_{c r} / q R_{0}$, which is the same in Refs. 17 and 25.

The situation is dramatically changed for the higher electron temperature $\tau_{e} \geq 1.5$ and the respective value of the hot ion temperature $T_{h} / T_{i} \approx 15 \tau_{e} / 2$. In this case, the starting normalized geodesic frequency begins to be lower, due to the changing sign of the hot ion correction to the dispersion $\Lambda\left(\lambda_{0}\right)<0$, the standard GAM frequency starts to diminish, and the mode begins to be unstable below $\Omega_{\text {uplim }}$. Then, two EGAM branches appear with higher frequency for $n_{h}>n_{h, \mathrm{cr}}$, and both appeared modes are stable. The mode splitting happens at the frequency $\Omega_{\text {splt }}^{2}=\left(7 / 2+2 \tau_{e}\right) q^{2} T_{i} / T_{h} \approx 4\left(3-\lambda_{0}\right.$ $\left.+\sqrt{3+\lambda_{0}^{2}-3 \lambda_{0}}\right)\left(1-\lambda_{0}\right) /\left(2-\lambda_{0}\right)$ when the classical GAM frequency coincides with the zero condition for the hot ion current $\operatorname{Im} J_{h} E_{1}^{*}=0$. Similar mode splitting into the stable GAM and the respective unstable EGAM driven by parallel NB injection for $n_{h} \geq 7 \%, q=3$, and $\tau_{e}=1$ is calculated in Ref. 27. However, we found that threshold may be substantially modified by the oblique injection to $n_{h} \approx 2 \%$ and the mode properties are changed, which means that the GAM moves to low frequency and begins to be unstable and higher frequency EGAM becomes stable for $\tau_{e}>1.5$.

Analyzing the distribution of the emitted power shown in Fig. 1 and in Eq. (8b), we conclude that the GAM instability is easily driven at lower pitch angles $\sqrt{1-\lambda_{0}}<0.5$, which is only limited by the region of trapped particles $\left(1-\lambda_{0}>\varepsilon\right)$. The additional limitations $\lambda_{0}^{2},\left(1-\lambda_{0}\right)^{2} \gg \Delta^{2}$ are used in Eq. (8), which is obtained in an asymptotic limit. In this case, the GAM frequency shown in Figs. 4 and 5 is substantially diminished from the standard values $\Omega_{h G A M}$ $=\sqrt{7 / 2+2 \tau_{e}} v_{T i} q / v_{T h} \approx 2-2.4$ to $1.2-1.3$. Similar frequency reduction has been also observed in the experiments. $^{10-13}$ It should be noted that our results are numerically different from that published ${ }^{15-17}$ earlier due to the thermal distribution of the bump at the critical velocity.

\section{B. GAM instability driven by rotation}

In an experimental series with NB heating, ${ }^{11-14}$ the GAM instability appears after the electron temperature saturation. This may happen before the bump density accumulation by the beam, which occurs after some time delay of the order of the slowing down time ${ }^{20} t_{\mathrm{sl}} \approx 6.3 \cdot 10^{8} A T_{e}^{3 / 2} /$ $Z_{e f} n_{0} \ln \Lambda$, which is approximately about $30-40 \mathrm{~ms}$ for standard plasmas. It means that the bump hot ions may have very low density. In this case of $n_{h} \Lambda\left(\lambda_{0}\right) \ll 1$, ignoring small imaginary part $\operatorname{Im}\left\langle j_{r}^{h} E_{1}^{*}\right\rangle$ in Eq. (8b), the respective decrement or increment can be calculated in the form

$$
\begin{aligned}
\gamma \approx & \sqrt{\frac{\pi}{2}} \frac{v_{T i} q}{2 R_{0}}\left\{4 \tau_{e} \frac{v_{0 e} v_{0 i}^{*}}{\Omega^{2}}-\left[\frac{\Omega^{4}}{2}+\Omega^{2}\left(2 \tau_{e}+1\right)\right]\right. \\
& \times \exp \left(-\frac{\Omega^{2}}{2}\right)-\frac{n_{h}\left(2-\lambda_{0}\right) \Omega^{5} v_{T i}^{5}}{24\left(1-\lambda_{0}\right)^{5 / 2} v_{T h}^{5}}\left(\frac{\left(2-\lambda_{0}\right) \Omega^{2} v_{T i}^{2}}{\left(1-\lambda_{0}\right) v_{T h}^{2}}-8\right) \\
& \left.\times \exp \left(-\frac{\Omega^{2} v_{T i}^{2}}{2\left(1-\lambda_{0}\right) v_{T h}^{2}}\right)\right\}
\end{aligned}
$$

where $\Omega \approx \omega_{G A M} R q / v_{T i}$ is the respective normalized GAM frequency. Naturally, it is possible that modifications of the basic plasma parameters may drive the instability of the upper GAM branch, which has small dissipation. Furthermore, to discuss the difference between co/counter NB injections on GAM stability in experiments, ${ }^{1-14}$ we have to take into account the asymmetric terms produced by the $E_{s}$-component in $\operatorname{Re} J_{\Sigma} E_{1}^{*}$. The co/counter NB effect does not appear directly in Eq. (1); it appears indirectly in radial current perturbations [Eqs. (2), (3), and (8)] through the plasma response to plasma species fluxes or diamagnetic terms via the $E_{c, s}$-parallel components of the electric field in density perturbations. These components couple the radial electric field in Eqs. (4) and (5) due to the quasi-neutrality condition. In this case, we have interplay ${ }^{14,28}$ (synergy effect) between the electron current, plasma flows, and drifts. Using $E_{s} \approx \mathrm{i} \sqrt{2 \pi} \frac{v_{0 e}}{\Omega} \frac{v_{T i} \tau_{e} E_{1}}{R_{0} \omega_{c i}}$, the cross-terms $v_{0 e} v_{0 i}^{*}$ appear in the imaginary and real parts of the $J_{\Sigma}$-current [Eq. (9)], modifying the GAM dispersion or increment, respectively, as it is shown in Eq. (12), where the $v_{0 e} v_{0 i}^{*}$-term is negative for co-injection and positive for the counter injection, which coincides with the electron velocity of the current. We also note that the synergetic effect similar to cold rotating ions may be produced by the hot bump ions for GAM frequencies $\Omega^{2}>4\left(1-\lambda_{0}\right) v_{T h}^{2} / v_{T i}^{2}$, and the detailed analyses will be published elsewhere.

Furthermore, estimating the Landau damping maximum at $\Omega_{\text {Landau }} \approx 2 \sqrt{1+1 / 2\left(1+\tau_{e}\right)}$, which begins to drop exponentially for the upper geodesic branch $\Omega>q \sqrt{7 / 2+2 \tau_{e}}$, we find that the instability may occur for $q \geq 2.2$ and $\tau_{e} \geq 1.1$, when $v_{0 e} v_{0 i}^{*} \geq 0.8 \cdot 10^{2}$. The electron current velocity may have the value $V_{0 e} \geq 0.1 v_{T e}$ in ohmic ${ }^{8}$ and counter NBI discharges, ${ }^{13,14}$ the dimensionless diamagnetic frequency is estimated $\Omega_{i}^{*} \approx 0.05, \eta_{i} \approx 1.5-2$, and the instability threshold may have been overcome, even though the plasma rotation is not measured in these discharges. It should be noted that the accumulated bump 
momentum (order of $n_{h} n_{0} V_{0} \sqrt{1-\lambda_{0}}$ ) begins to be transferred to the main plasma after $t_{\mathrm{sl}}$-time delay due to ion-ion collisions, ${ }^{20}$ increasing or reducing the plasma rotation by this value. In this case, the emitted/dissipated power in Eq. (11) should be corrected with the additional velocity $V_{\mathrm{ad}} \approx n_{b} V_{c r} \sqrt{1-\lambda_{0}} t_{D i} / t_{s l}$, where $t_{D i}$ is the ion confinement time.

\section{GAM formation and orbit width effect (FOW)}

Here, our study is limited by zero FRL and FOW effects, ${ }^{17,27}$ which are responsible for eigenmode formation ${ }^{28}$ defined by the respective $k_{r} \rho_{L}$-parameter, and an additional FOW dissipation ${ }^{23}$ begins to be strong for $k_{r} \rho_{L} q>0.1, \tau_{e}$ $\approx 1-2$ in comparison to Landau damping. Typically, the formation of the EGAM eigenmode structure is assumed that to be at maxima (or minima) of the geodesic continuum, ${ }^{28,29}$ which depends on the radial distribution of the energetic ion density and electron temperature in the radius. The EGAM continuum dependence on the parameters may be approximated by the formula $\omega_{E G A M} \approx 2 V_{c r} / R q \sqrt{3 n_{h}}$ adapted to the data shown in Figs. 4 and 5 in the interval $2 \%<n_{h}<6 \%$. The continuum maximum/minimum depends on $\partial \ln T_{e} /$ $\partial \ln n_{h}$, and it may easily appear at $q=q_{\min }$ in specific tokamak discharges with shear reversal and preferentially electron heating by NB injection. ${ }^{12,13}$ In this case, the FOW parameter may be small $k_{r} \rho_{L} q<0.1$ for $q_{\text {min }}<3$. In Fig. 6 , the frequencies of geodesic continua modified by energetic ions are schematically reproduced for DIII-D parameters; ${ }^{10,12} \lambda_{0}=0.6, T_{O e}=1.8 \mathrm{keV}, \tau_{e}=1.4$, and $T_{h} / T_{e}=15 / 2$ with respective profiles $T_{\alpha}=T_{0 \alpha}\left(1-x^{2}\right)^{2.5}, x=r / a$, and $q=2 x\left(4 x^{2}-2 x-1\right)+4.4$, where $q_{\min }=3.4$ at $x=0.5$ and the hot ion concentrations are simulated for the on-axis beam injection, ${ }^{21}$ as an example, by Gaussian radial distributions $\propto n_{0 b} \exp \left(-2 r^{2} / a^{2}\right)$ with $n_{0 b}=0 \%, 2.5 \%$, and $6 \%$. We note that the lowest modes ( $\mathrm{f}=22$ and $29 \mathrm{kHz}$ ) related to $n_{0 b}$ $=2.5 \%$ and $6 \%$, which are marked by solid and dash-dotted lines, may be unstable according to dissipative limitations presented in Figs. 3 and 4.

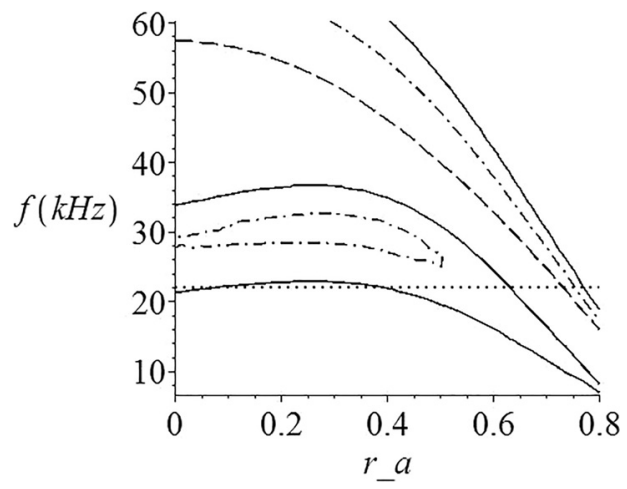

FIG. 6. Plot of the GAM and EGAM continuum frequency $[\mathrm{kHz}]$ branches for $T_{e 0}=2 \mathrm{keV}, \tau_{e}=1.4, T_{h} / T_{e}=7.5$, and $q_{\min }=3.4$ at $r / a=0.5$ as a function of the normalized minor radius for different $\mathrm{NB}$ concentrations ( $n_{0 b}=0 \%$-dashed, $n_{0 b}=2.5 \%$-dash-dotted, and $n_{h}=6 \%$-solid line), and the horizontal line presents an expected eigenmode frequency. ${ }^{28,29}$

\section{SUMMARY}

As mentioned in the Introduction, we first note that the new features in our model are to consider the situation in which the GAM phase resonance may appear at the critical velocity, where the energetic ions form the bump which begins to be substantially diffused/scattered which is also considered in detail the difference in the physics of the modes driven by the NB injection. Specifically, we found limitations (due to Landau damping in hot and cold ions) on the neutral beam density to drive the GAM instability, in oblique injection, at the critical ion circulation frequency $\omega_{G}<2 \sqrt{2\left(1-\lambda_{0}\right) /\left(2-\lambda_{0}\right)} v_{T h} / q R_{0}$, where there is a maximum of the distribution. Actually, the slowing down model used in Refs. 15 and 16 is not valid at the circulation frequency, where the maximum of the hot ion distribution has to be located, and the hot beam model discussed in Refs. 17 and 27 is related only to parallel injection. On the other hand, the cold beam model discussed in Refs. 18 and 19 does not include the effect of Landau damping in hot and cold ions, which produces a strong limitation on the geodesic instability due to the condition $T_{h} \ll E_{c r}$. All these effects are taken into account in this work.

Finally, we may conclude the following:

In the approximation of the hot bump-on-tail model, the effect of a minor concentration of energetic ions produced by oblique unbalanced NB injection on the GAM spectrum is successfully evaluated using analytical solutions of the drift kinetic equation when basic plasma particles are treated in the fluid model in the limit of the large safety factor.

It is demonstrated that the GAM-EGAM splitting (bifurcation) of the unstable mode appears for substantially lower neutral beam densities in comparison to the value observed for parallel injection, as shown in Refs. 17 and 27. The effect appears due to an additional drive by the inhomogeneous dependence on the pitch angle distribution.

The regions of the GAM instability characterized by the negative dissipated power density are found below the frequency $\quad \omega_{\lim }=2 \sqrt{2\left(1-\lambda_{0}\right) /\left(2-\lambda_{0}\right)} v_{T h} / q R_{0} \quad$ and shown in Figs. 1-4. Two kinds of unstable modes are discussed, which are the standard GAM and energetic GAM shown in Fig. 5. The EGAM instability may be only excited above the critical energetic ion density that depends on the relationship between the safety factor parameter, pitch angle, and electron temperature, and the results are used to reproduce the EGAM observations in DII-D experiments, which are shown in Fig. 6. The GAM instability may be driven before the bump-on-tail formation, but after electron heating, it results from the crossterm of the electron/ion fluxes and diamagnetic effect additionally induced by the NB injection. In this case, the counter NBI has the preference for GAM excitation in comparison to the co-injection.

\section{ACKNOWLEDGMENTS}

This work was supported by the CAPES student grant and CNPq (National Council of Scientific and Technological Development) with Contract No. 306757/2015-0, Brazil. 
${ }^{1}$ V. B. Lebedev, P. N. Yushmanov, P. H. Diamond, S. V. Novakovskii, and A. I. Smolyakov, Phys. Plasmas 3, 3023 (1996).

${ }^{2}$ P. H. Diamond, S.-I. Itoh, K. Itoh, and T. S. Hahm, Plasma Phys. Controlled Fusion 47, R35 (2005).

${ }^{3}$ K. Hallatschek and D. Biskamp, Phys. Rev. Lett. 86, 1223 (2001).

${ }^{4}$ G. D. Conway, Plasma Phys. Controlled Fusion 50, 124026 (2008).

${ }^{5}$ G. D. Conway, C. Angioni, F. Ryter, P. Sauter, and J. Vicente, Phys. Rev. Lett. 106, 065001 (2011).

${ }^{6}$ W. W. Heidbrink, Phys. Plasmas 15, 055501 (2008).

${ }^{7}$ L. G. Askinazi, A. A. Belokurov, V. V. Bulanin, A. D. Gurchenko, E. Z. Gusakov, T. P. Kiviniemi, S. V. Lebedev, V. A. Kornev, T. Korpilo, S. V. Krikunov, S. Leerink, M. Machielsen, P. Niskala, A. V. Petrov, A. S. Tukachinsky, A. Yu. Yashin, and N. A. Zhubr, Plasma Phys. Controlled Fusion 59, 014037 (2017).

${ }^{8}$ A. V. Melnikov, L. G. Eliseev, S. V. Perfilov, S. E. Lysenko, R. V. Shurygin, V. N. Zenin, S. A. Grashin, L. I. Krupnik, A. S. Kozachek, R. Yu. Solomatin, A. G. Elfimov, A. I. Smolyakov, and M. V. Ufimtsev, Nucl. Fusion 55, 063001 (2015).

${ }^{9}$ H. L. Berk, C. J. Boswell, D. Borba, A. C. A. Figueiredo, T. Johnson, M. F. F. Nave, S. D. Pinches, S. E. Sharapov, and JET Contributors, Nucl. Fusion 46, S888 (2006).

${ }^{10}$ R. Nazikian, G. Y. Fu, M. E. Austin, H. L. Berk, R. V. Budny, N. N. Gorelenkov, W. W. Heidbrink, C. T. Holcomb, G. J. Kramer, G. R. McKee, M. A. Makowski, W. M. Solomon, M. Shafer, E. J. Strait, and M. A. Van Zeeland, Phys. Rev. Lett. 101, 185001 (2008).

${ }^{11}$ G. R. McKee, D. K. Gupta, R. J. Fonck, D. J. Schlossberg, M. W. Shafer, and P. Gohil, Plasma Phys. Controlled Fusion 48, S123 (2006).

${ }^{12}$ G. Wang, W. A. Peebles, T. L. Rhodes, M. E. Austin, Z. Yan et al., Phys. Plasmas 20, 092501 (2013).
${ }^{13}$ G. Matsunaga, K. Kamiya, K. Shinohara, N. Miyato, A. Kojima, and A. Bierwage, in 39th EPS Conference \& 16thICPP, July 2012, Stockholm/ Sweden, EPS Abstract Series Stockholm/Sweden (2012), p. P2.062.

${ }^{14}$ F. Camilo de Souza, A. G. Elfimov, R. M. O. Galvão, J. Krbec, J. Seidl, J. Stöckel, M. Hron, J. Havlicek, and K. Mitosinkova, Phys. Lett. A 381, 3066 (2017).

${ }^{15}$ G. Y. Fu, Phys. Rev. Lett. 101, 185002 (2008).

${ }^{16}$ Z. Qiu, F. Zonca, and L. Chen, Plasma Phys. Controlled Fusion 52, 095003 (2010).

${ }^{17}$ D. Zarzoso, X. Garbet, Y. Sarazin, R. Dumont, and V. Grandgirard, Phys. Plasmas 19, 22102 (2012).

${ }^{18}$ Z. S. Qu, M. J. Hole, and M. Fitzgerald, Phys. Rev. Lett. 116, 095004 (2016).

${ }^{19}$ Z. S. Qu, M. J. Hole, and M. Fitzgerald, Plasma Phys. Controlled Fusion 59, 055018 (2017).

${ }^{20}$ T. H. Stix, Plasma Phys. 14, 367 (1972).

${ }^{21}$ W. W. Heidbrink, M. A. Van Zeeland, M. E. Austin, E. M. Bass, K. Ghantous, N. N. Gorelenkov, B. A. Grierson, D. A. Spong, and B. J. Tobias, Nucl. Fusion 53, 093006 (2013).

${ }^{22}$ A. I. Smolyakov and X. Garbet, Phys. Plasmas 17, 042105 (2010).

${ }^{23}$ M. Artun and W. M. Tang, Phys. Plasmas 1, 2682 (1994).

${ }^{24}$ A. G. Elfimov, A. I. Smolyakov, and R. M. O. Galvão, Phys. Lett. A 378, 800 (2014).

${ }^{25}$ H. Sugama and T.-H. Watanabe, J. Plasma Phys. 72, 825 (2006).

${ }^{26} \mathrm{M}$. Abramowitz and I. A. Stegun, Handbook of Mathematical Functions (Dover, New York, 1972).

${ }^{27}$ J.-B. Girardo, D. Zarzoso, R. Dumont, X. Garbet, Y. Sarazin, and S. Sharapov, Phys. Plasmas 21, 092507 (2014).

${ }^{28}$ A. G. Elfimov, Phys. Lett. A 378, 3533 (2014).

${ }^{29}$ S. I. Itoh, K. Itoh, M. Sasaki, A. Fujisawa, T. Ido, and Y. Nagashima, Plasma Phys. Controlled Fusion 49, L7-L10 (2007). 